Paper

\title{
Multiple basins of consistency in noise-driven dynamical system
}

\author{
Hoipang Yip $^{1}$ and Atsushi Uchida ${ }^{2 a)}$ \\ ${ }^{1}$ Department of Electronics and Computer Systems, Takushoku University, \\ 815-1 Tatemachi, Hachioji, Tokyo 193-0985, Japan \\ ${ }^{2}$ Department of Information and Computer Sciences, Saitama University, \\ 255 Shimo-okubo, Sakura-ku, Saimata City, Saimata 338-8570, Japan \\ a) auchida@mail.saitama-u.ac.jp
}

Received January 24, 2014; Revised May 5, 2014; Published October 1, 2014

\begin{abstract}
We numerically investigate multiple basins of consistency in the Mackey-Glass model driven repeatedly by a colored noise signal. The Mackey-Glass model is modified to include the saturation effect for the observation of multiple basins of consistency. Consistent response outputs are obtained for strong drive strength, whereas no consistency is observed for small drive strength. For intermediate drive strength, more than one type of consistent response waveforms can be observed, known as multiple basins of consistency. The regions of multiple basins are entangled in the phase space. The number of multiple basins of consistency depends on the drive strength and the saturation threshold in the Mackey-Glass model. Our findings are confirmed experimentally by using an electronic circuit of the Mackey-Glass model with a colored-noise drive signal.
\end{abstract}

Key Words: consistency, synchronization, noise, multiple basin of consistency, electronic circuit

\section{Introduction}

Consistent behavior to a repeated input signal has been observed in many nonlinear dynamical systems. Consistency is defined as reproducibility of the output of a nonlinear system driven repeatedly by a complex signal. Consistency has been considered as a common concept observed in various nonlinear dynamical systems in interdisciplinary research fields. Experimental observation of consistency has been reported in neuronal networks and laser systems [1-3]. Numerical simulations of consistency have also been investigated in the context of common-signal-induced synchronization [48]. The concept of consistency can be useful for engineering applications to noninvasive testing [9], implementation of physical one-way function [10], and implementation of reservoir computing $[11,12]$.

The concept of consistency is related to generalized synchronization [13, 14]. Generalized synchronization is defined as a functional relationship between the drive and response temporal waveforms. On the contrary, consistency is defined as reproducibility of a nonlinear dynamical system driven by a repeated complex signal. Generalized synchronization is considered as "relationship" between coupled dynamical systems, whereas consistency can be considered as "stability" for a solitary driven dynam- 
ical system [3]. Many papers on generalized synchronization use the auxiliary system method [13] to measure synchronization, however, the auxiliary system method only measures stability. In this sense, consistency viewpoint could be more widely applied for many nonlinear dynamical systems driven by a complex signal.

The concept of multiple basins of consistency has been proposed [15], where more than one consistent output can be obtained from a nonlinear dynamical system driven by a repeated signal. Multiple basins of consistency can be interpreted as the existence of multiple attractors for undriven dynamical systems, and the attractors that are modified by the drive signal are still stable. The concept of multiple basins of consistency may be related to multistable phenomena in a driven system, such as Necker cube in human perception. Multiple basins of consistency have been observed numerically in the driven Lorenz system for weak drive strength [15]. An experimental observation of multiple basins of consistency has been reported in a Mackey-Glass electronic circuit [16]. However, the condition for the appearance of multiple basins of consistency has not been investigated in detail.

In this study we numerically investigate multiple basins of consistency in the Mackey-Glass model driven repeatedly by a colored noise signal. We introduce a modified Mackey-Glass model with a saturation effect to observe multiple basins of consistency. We quantitatively investigate the degree of consistency when the drive signal strength is changed. The number of multiple basins of consistency is investigated for different parameter regions. We also experimentally observe multiple basins of consistency with an electronic circuit of the Mackey-Glass model.

\section{Numerical simulation}

\subsection{Model}

We used the Mackey-Glass model [17] with an external drive signal. The equation of the Mackey-Glass model is modified with a saturation effect in order to include the limitation of the maximum gain of electronic amplifiers in a Mackey-Glass electronic circuit. The saturation effect is crucial to reproduce our experimental results, as described in Section 3. The modified Mackey-Glass model is described as follows.

$$
\begin{gathered}
\frac{d V(t)}{d t}=\frac{a c M(t)}{1+[c M(t)]^{n}}-b V(t) \\
M(t)= \begin{cases}\kappa D(t)+V(t-\tau) & \left(-S_{v}<\kappa D(t)+V(t-\tau)<S_{v}\right) \\
S_{v} & \left(\kappa D(t)+V(t-\tau) \geq S_{v}\right) \\
-S_{v} & \left(\kappa D(t)+V(t-\tau) \leq-S_{v}\right)\end{cases}
\end{gathered}
$$

where $V(t)$ is the output of the Mackey-Glass model, $a$ is the height of the nonlinear function, $b$ is the decay rate, $\tau$ is the delay time, $n$ is the shape of the nonlinear function, $c$ is the feedback strength, $D(t)$ is the colored-noise drive signal, $\kappa$ is the drive strength, $M(t)$ is the mixed signal between the drive and feedback signals, and $S_{v}$ is the saturation threshold value. The mixed signal $M(t)$ is clipped at $\pm S_{v}$ due to the gain saturation effect of electronic amplifiers, as seen in Eq. (2). The parameter values are set as follows: $a=2.0, n=10.0, \tau=5.0, b=1.0, c=1.0, \kappa=0.6$, and $S_{v}=1.6$. We used an exponentially correlated colored-noise waveform generated using an Ornstein-Uhlenbeck process [18] for the drive signal $D(t)$, such that $\langle D(t) D(t+\tau)\rangle=\left(2 A / \tau_{c}\right) \exp \left(-\tau / \tau_{c}\right)$, where $A$ is the noise strength and $\tau_{c}$ is the correlation time of the colored noise. The correlation time $\tau_{c}=3.3$ of the colored noise is used in our simulations.

\subsection{Numerical result}

We numerically calculated temporal waveforms of the Mackey-Glass model driven by a same colorednoise signal. We repeated the calculation of temporal waveforms for different initial conditions with the same colored-noise drive signal. Figure 1 shows the temporal waveforms of the drive signal and four corresponding response signals (called Response 1,2,3, and 4) starting from different initial conditions. We also show the correlation plots of the temporal waveforms between Response 1 and 2 , 


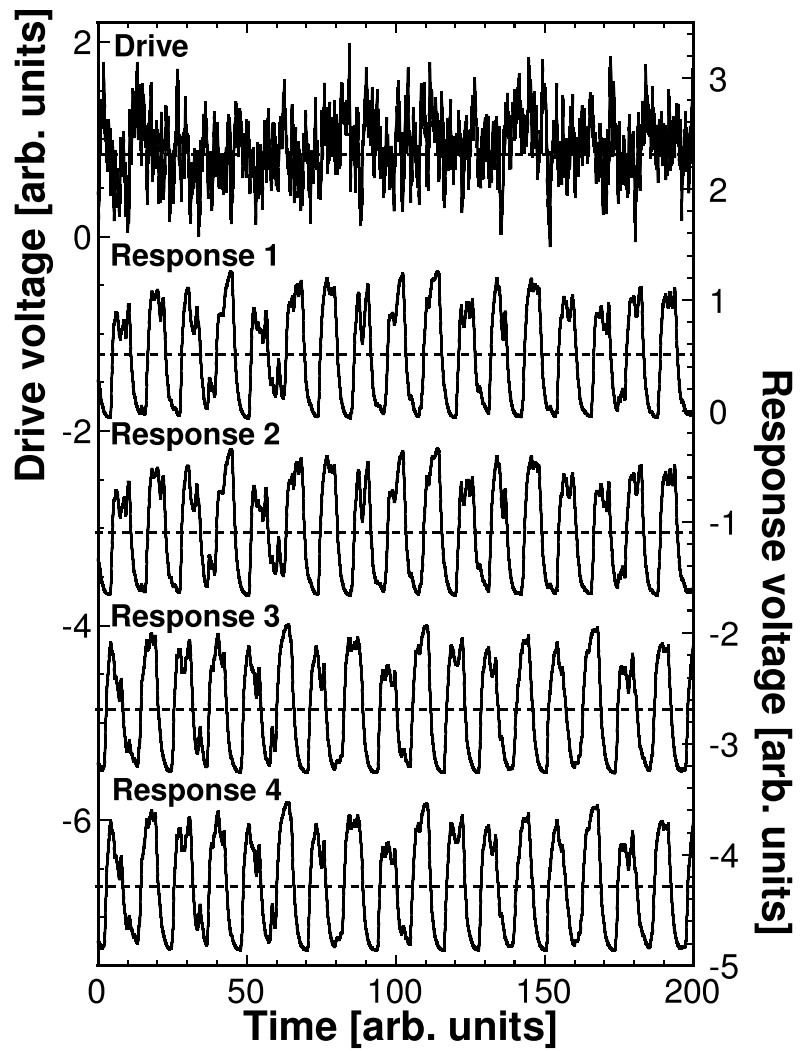

Fig. 1. Numerical result of the temporal waveforms of the colored-noise drive signal and the corresponding four response signals. Consistent outputs are obtained between Response 1 and 2, and between Response 3 and 4 . The voltages of the temporal waveforms are shifted for clarity. The dotted lines indicate the common voltage of the temporal waveforms.

(a)

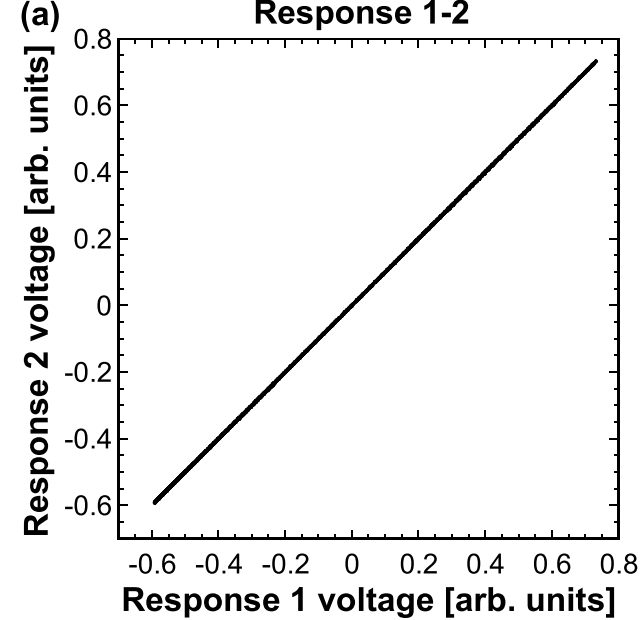

(b)

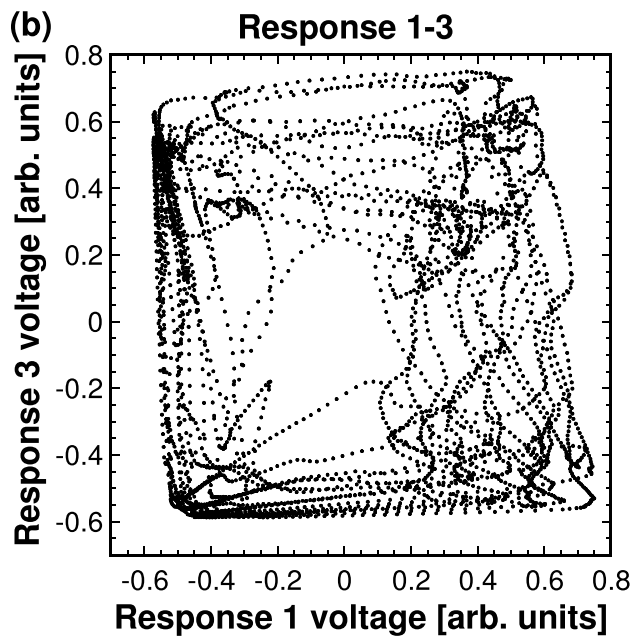

Fig. 2. Numerical result of the correlation plots (a) between Response 1 and 2 , and (b) between Response 1 and 3.

and between Response 1 and 3 in Fig. 2. The temporal waveforms of Response 1 and 2 are identical, i.e., consistent outputs are observed. Consistency is also observed between the temporal waveforms of Response 3 and 4. However, other pairs of the four temporal waveforms look different (e.g., Response 1 and 3). Two types of consistent response waveforms are observed even though the Mackey-Glass model is driven by the same colored-noise drive signal. This result shows an evidence of multiple basins of consistency.

To investigate the characteristics of consistency quantitatively, we introduce a measure of consistency $C$ as the average cross-correlation between a pair of response waveforms obtained from the 

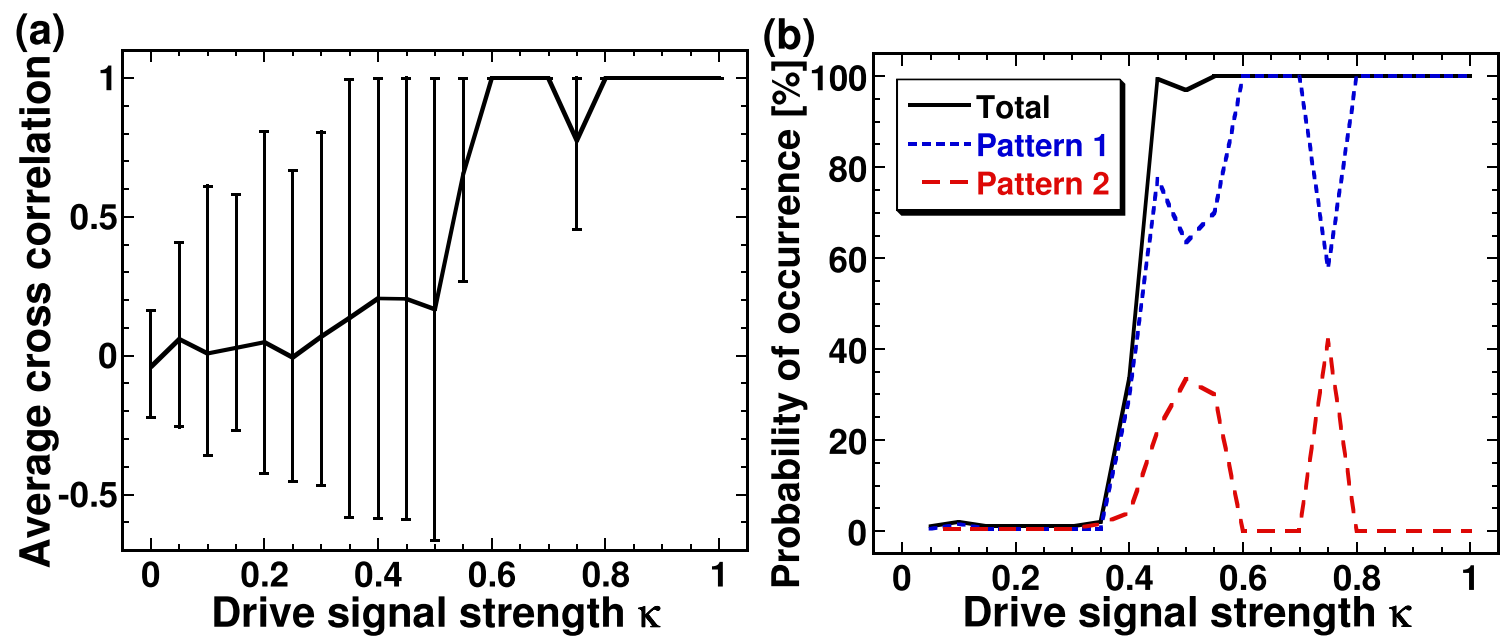

Fig. 3. Numerical results of (a) the average cross correlation between a pair of the response waveforms with error bars and (b) the probability of the occurrence of the two consistent temporal waveforms (two patterns). (a) The regions with large error bars and the maximum $C=1$ correspond to multiple basins of consistency. (b) The two consistent temporal patterns are observed at $\kappa=0.45,0.50,0.55$, and 0.75 .

dynamical system driven by a repeated drive signal [15],

$$
C=\frac{2}{N(N-1)} \sum_{i=1}^{N} \sum_{j=1(j>i)}^{N}\left[\frac{<\left(x_{i}(t)-\bar{x}_{i}\right)\left(x_{j}(t)-\bar{x}_{j}\right)>}{\sigma_{i} \sigma_{j}}\right],
$$

where $x_{i, j}(t)$ are the temporal waveforms of the Response $i$ and $j$ signals, $\bar{x}_{i, j}$ are the mean values of the temporal waveforms of the Response $i$ and $j$, and $\sigma_{i, j}$ are the standard deviations of the temporal waveforms of the Response $i$ and $j$. The angle brackets denote time averaging.

Figure 3(a) shows the average consistency value $C$ for 100 response waveforms as the drive signal strength $\kappa$ is increased. The error bars indicate the maximum and minimum values of the cross correlation between two response waveforms. The average cross-correlation values $C$ approaches 1 as $\kappa$ is increased. The error bars also become small for large $\kappa$. However, there are some regions where the maximum value of $C$ is 1 and large error bars are observed (e.g., for $\kappa=0.75$ ). This region corresponds to multiple basins of consistency, because the response waveforms are clustered and large $C$ is obtained for the corresponding pairs of the response waveforms, whereas small $C$ is observed for the different pairs of the response waveforms.

Figure 3(b) shows the probability of the occurrence of the two consistent temporal waveforms (called pattern 1 and 2) as $\kappa$ is increased. We found that the two consistent patterns are obtained for $\kappa=0.45,0.50,0.55$, and 0.75 . The regions for multiple basins of consistency are observed at intermediate strength of $\kappa$. The occurrence of the pattern 1 and 2 varies for different $\kappa$, and the probability of the occurrence of the pattern 1 changes as $\kappa$ is increased for the regions of the multiple basins of consistency. We interpret that multiple basins of consistency derives from multi-stability of the Mackey-Glass model with time-delayed feedback, where more than one attractor coexist even in the presence of the drive signal.

We also numerically investigated the basin structure in the phase space. We calculated temporal waveforms starting from 6000 different initial conditions on the original chaotic attractor. We classified the response temporal waveforms into two groups of consistent waveforms. We then plotted the sets of initial conditions on the phase space. We used a time-delayed embedding method to construct a chaotic attractor in the high-dimensional phase space, which consists of $V(t), V(t+\tau), V(t+2 \tau)$, and so on. We created the two-dimensional projection $(V(t)$ versus $V(t+\tau))$ of the re-constructed high-dimensional chaotic attractor. Figure 4 shows the basins of consistency plotted on the twodimensional projection of the chaotic attractor for the Mackey-Glass model driven by a colored noise signal. Red and blue colors indicate the two types of basins (pattern 1 and 2). The two basins are 


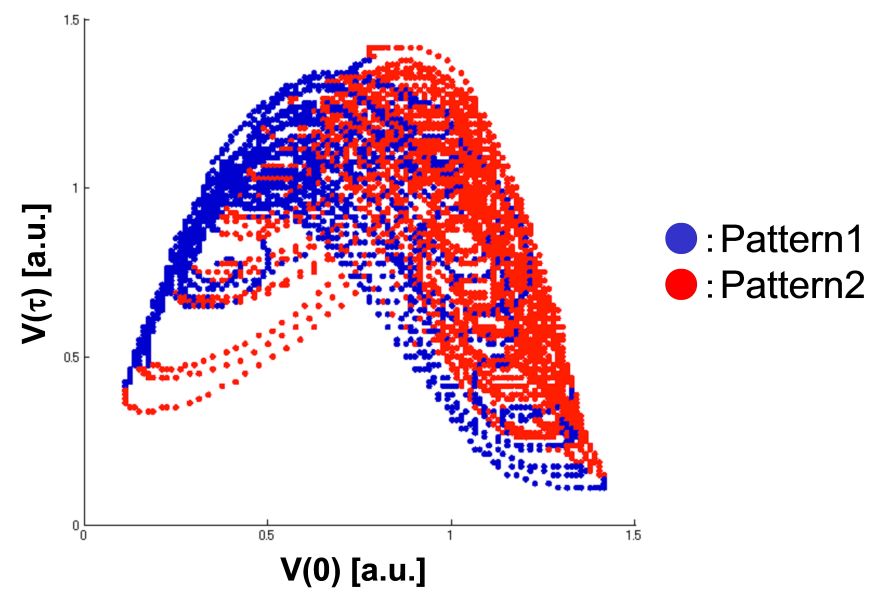

Fig. 4. Numerical result of multiple basins of consistency plotted on the twodimensional projection of the chaotic attractor for the Mackey-Glass model. The plots with blue and red colors correspond to basin 1 and 2, respectively.

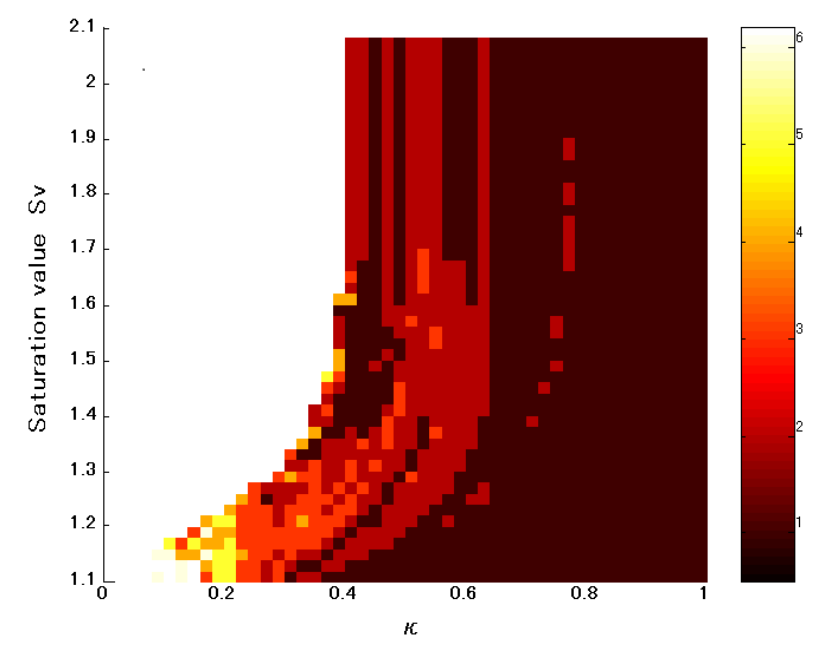

Fig. 5. Numerical result of the two-dimensional map for the number of multiple basins of consistency as functions of the drive singal strength $\kappa$ and the saturation threshold value $S_{v}$. White region, no consistency; Dark brown region, single basin of consistency. Other colors, multiple basins of consistency.

entangled and there are no clear basin boundaries. The complicated basin structure indicates that it is important to evaluate consistency carefully for different initial conditions in the presence of multiple basins. Note that the Mackey-Glass model with time-delayed feedback has high dimensionality and this basin structure is projected on the two-dimensional phase space. This riddled basin structure results from the high dimensionality of the chaotic attractor.

Next we investigated the parameter dependence of the number of multiple basins of consistency in numerical simulations. We calculated 100 temporal waveforms of the response signals and classified into consistent attractors. The number of coexisting attractors is counted as the number of the multiple basins of consistency for different parameter values. Figure 5 shows the number of multiple basins of consistency as functions of the drive signal strength $\kappa$ and the saturation threshold value $S_{v}$. The white regions in Fig. 5 correspond to no consistency, and dark brown regions correspond to a single basin of consistency. The regions for multiple basins of consistency exist between these two regions for intermediate $\kappa$ (light brown and yellow regions). For large $S_{v}$, only two basins of consistency are obtained. On the contrary, more than two basins of consistency can be observed for small $S_{v}$ and intermediate $\kappa$. Five coexisting attractors can be distinguished at maximum in our simulation. The saturation effect of the Mackey-Glass model strongly affects the number of multiple basins of consistency. The saturation threshold determines the maximum peak value of chaotic temporal waveforms. The peak values of chaotic waveforms become smaller as the saturation 


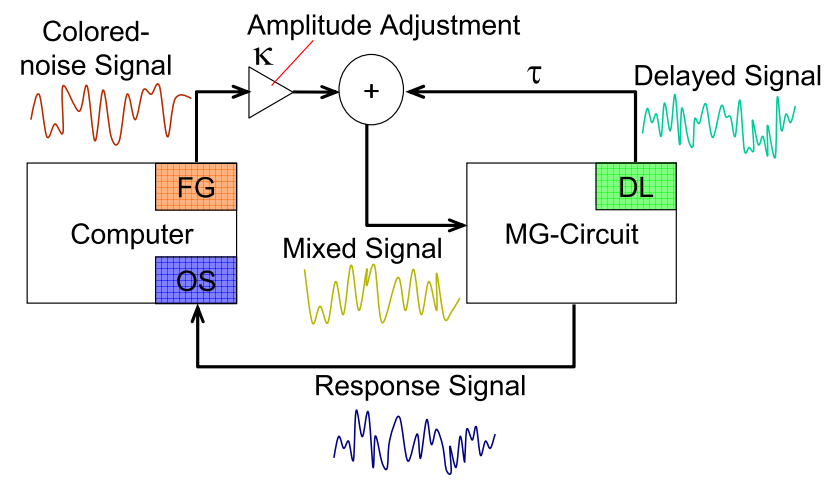

Fig. 6. Experimental setup for the Mackey-Glass electronic circuit with timedelayed feedback driven by a colored-noise signal. DL, delay line; FG, function generator; OS, oscilloscope.

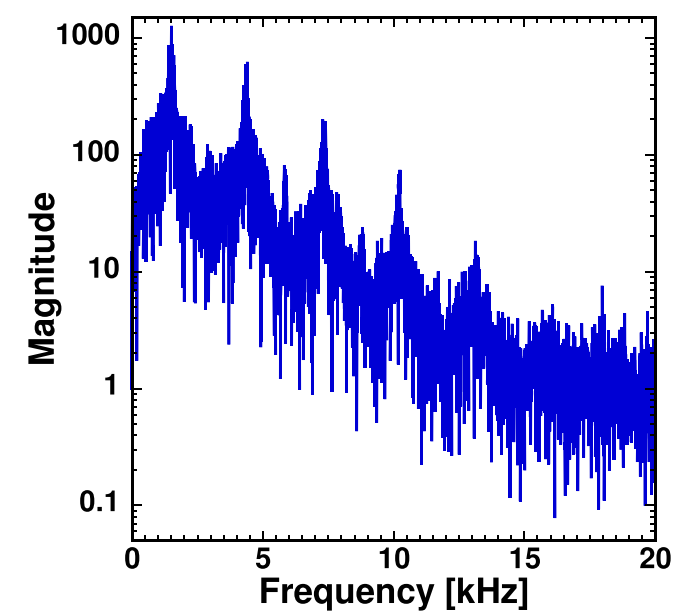

Fig. 7. Frequency spectrum of chaotic temporal waveform in the MackeyGlass electronic circuit without colored-noise drive signal. The center frequency is $1.5 \mathrm{kHz}$.

threshold is decreased. The temporal waveforms thus look like periodic oscillations for small satulation threshold values. We speculate that a large number of nearly periodic temporal waveforms can coexist with phase shift for small saturation threshold values.

\section{Experiment}

\subsection{Experimental setup}

To confirm our numerical observation, we implemented a Mackey-Glass electronic circuit in experiment [16]. Figure 6 shows our experimental setup for the observation of consistency [16]. We used a Mackey-Glass electronic circuit with time-delayed feedback as a nonlinear dynamical system [19-21]. The circuit consists of two field-effect transistors (2SJ103 and 2SK30A, Toshiba) for the nonlinear part, three electronic amplifiers, an RC filter, and a delay line. 50 pairs of capacitors $(10 \mathrm{nF})$ and inductors $(4.7 \mathrm{mH})$ are combined to generate time delay. The total delay time is $318 \mu \mathrm{s}$, corresponding to half period of the chaotic waveform [21].

Figure 7 shows the frequency spectrum of the chaotic temporal waveform in the Mackey-Glass electronic circuit. It is found that the center frequency of the chaotic temporal waveform is $1.5 \mathrm{kHz}$. We used an exponentially correlated colored-noise waveform generated using an Ornstein-Uhlenbeck process [18] as a drive signal. The inverse of the correlation time of the colored-noise drive signal is set to $f_{c}=1.5 \mathrm{kHz}$ to match the center frequency of the chaotic temporal waveform in the Mackey-Glass model without colored-noise drive signal.

The colored noise signal is generated numerically and stored in a computer. The colored noise signal is reproduced by using a function generator. The amplitude of the colored noise signal is adjusted by using an amplifier and added with a time-delayed feedback signal of the chaotic electronic circuit at 


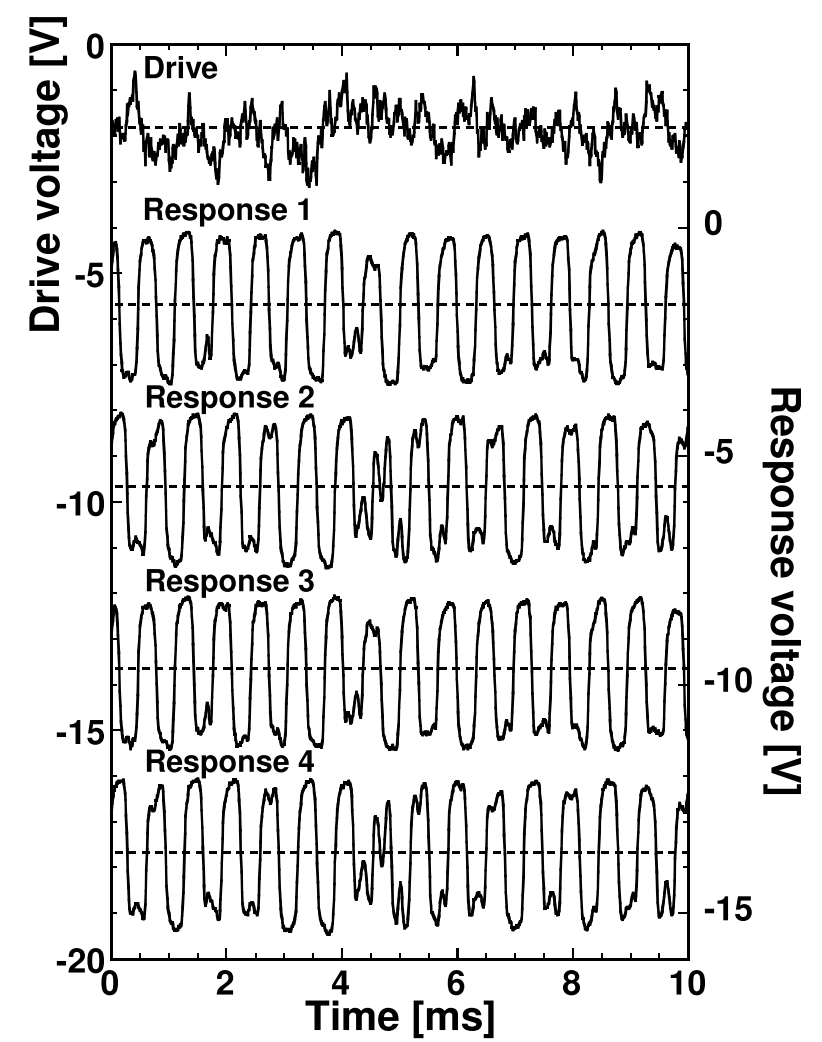

Fig. 8. Experimental result of the temporal waveforms of the colored-noise drive signal and the corresponding four response signals. Consistent outputs are obtained between Response 1 and 3, and between Response 2 and 4. The voltages of the temporal waveforms are shifted for clarity. The dotted lines indicate the common voltage of the temporal waveforms.

(a)

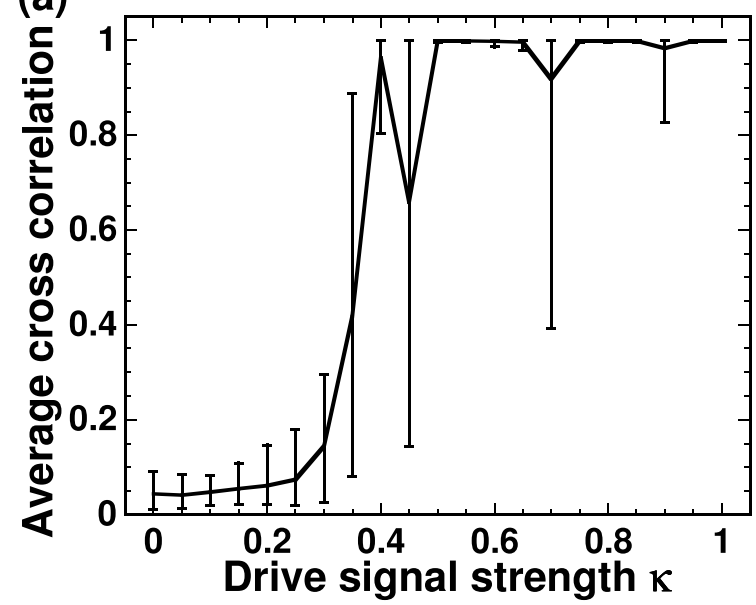

(b)

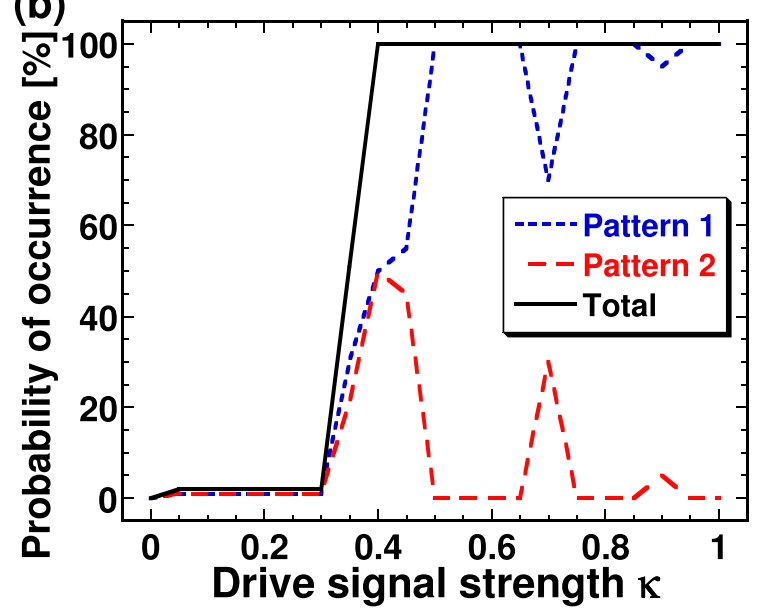

Fig. 9. Experimental results of (a) the average cross correlation between a pair of the response waveforms with error bars and (b) the probability of the occurrence of the two consistent temporal waveforms. (a) The regions with large error bars and the maximum $C=1$ correspond to multiple basins of consistency at $\kappa=0.40,0.45,0.70$, and 0.90 . (b) The two consistent temporal patterns are observed at $\kappa=0.40,0.45,0.70$, and 0.90 .

an adder circuit, as shown in Fig. 6. The mixed signal is sent to the nonlinear part of the electronic circuit. The voltage of the electronic circuit after the delay line is observed by a digital oscilloscope. The drive signal is repeatedly sent to the circuit and several response waveforms corresponding to the identical drive signal are detected. Consistency can be evaluated by calculating the cross correlation between a pair of the response waveforms obtained from the repeated drive signal (see Eq. (3)). 


\subsection{Experimental result}

Figure 8 shows the experimentally-obtained temporal waveforms of the drive signal and four corresponding response signals detected at different times. The temporal waveforms of Response 1 and 3 are consistent, and consistency is also observed between Response 2 and 4. On the contrary, other pairs of the four temporal waveforms look different (e.g., Response 1 and 2). This experimental result agrees well with our numerical result shown in Fig. 1.

Figure 9(a) shows the experimental result of the average consistency value $C$ for 20 response waveforms as the drive signal strength $\kappa$ is increased. The average cross-correlation value $C$ increases as $\kappa$ is increased and the error bars become small for large $\kappa$. The regions for multiple basins of consistency are found for $\kappa=0.40,0.45,0.70$, and 0.90 , with the maximum $C$ of 1 and large error bars. Figure 9(b) shows the experimental result of the probability of the occurrence of the two consistent temporal waveforms as $\kappa$ is increased. The regions for multiple basins of consistency are observed for intermediate values of $\kappa=0.40,0.45,0.70$, and 0.90. These experimental results of Figs. 9(a) and 9(b) are consistent with our numerical results shown in Figs. 3(a) and 3(b).

\section{Conclusion}

We have numerically investigated multiple basins of consistency in the Mackey-Glass model driven repeatedly by a colored-noise signal. The Mackey-Glass model is modified to include the saturation effect for the observation of multiple basins of consistency. Consistency is observed for strong drive strength, whereas no consistency is found for small drive strength. More than one type of response waveforms are observed for intermediate drive strength, known as multiple basins of consistency. The regions of the two basins are entangled in the phase space. The number of multiple basins of consistency is dependent of the drive strength and the saturation threshold. Our findings are confirmed by experimental observation of multiple basins of consistency in the electronic circuit of the MackeyGlass model. Multiple basins of consistency may be a general concept that can be observed in many nonlinear dynamical systems.

\section{Acknowledgments}

We acknowledge support from Grant-in-Aid for Young Scientists and Magagement Expenses Grants from the Ministry of Education, Culture, Sports, Science and Technology in Japan.

\section{References}

[1] Z.F. Mainen and T.J. Sejnowski, Science, vol. 268, p. 1503, 1995.

[2] A. Uchida, R. McAllister, and R. Roy, Phys. Rev. Lett., vol. 93, p. 244102, 2004.

[3] A. Uchida, Optical Communication with Chaotic Lasers, Applications of Nonlinear Dynamics and Synchronization, Wiley-VCH, Weinheim, 2012.

[4] R. Toral, C.R. Mirasso, E. Hernandez-Garcia, and O. Piro, Chaos, vol. 11, p. 665, 2001.

[5] C. Zhou and J. Kürths, Phys. Rev. Lett., vol. 88, p. 230602, 2002.

[6] J. Teramae and D. Tanaka, Phys. Rev. Lett., vol. 93, p. 204103, 2004.

[7] K. Nagai, H. Nakao, and Y. Tsubo, Phys. Rev. E, vol. 71, p. 036217, 2005.

[8] K. Yoshimura, I. Valiusaityte, and P. Davis, Phys. Rev. E, vol. 75, p. 026208, 2007.

[9] L. Moniz, T. Carroll, L. Pecora, and M. Todd, Phys. Rev. E, vol. 68, p. 036215, 2003.

[10] R. Pappu, B. Recht, J. Taylor, and N. Gershenfeld, Science, vol. 297, p. 2026, 2002.

[11] L. Appeltant, M.C. Soriano, G. Van der Sande, J. Danckaert, S. Massar, J. Dambre, B. Schrauwen, C.R. Mirasso, and I. Fischer, Nat. Commun., vol. 2, p. 468, 2011.

[12] L. Larger, M.C. Soriano, D. Brunner, L. Appeltant, J.M. Gutierrez, L. Pesquera, C.R. Mirasso, and I. Fischer, Opt. Express, vol. 20, p. 3241, 2012.

[13] H.D.I. Abarbanel, N.F. Rulkov, and M.M. Sushchik, Phys. Rev. E, vol. 53, p. 4528, 1996.

[14] A.S. Pikovsky, M. Rosenblum, and J. Kürths, Synchronization, Cambridge University Press, Cambridge, 2001.

[15] A. Uchida, K. Yoshimura, P. Davis, S. Yoshimori, and R. Roy, Phys. Rev. E, vol. 78, p. 036203, 2008. 
[16] H. Yip, S. Sano, A. Uchida, and S. Yoshimori, Proceedings of 2007 International Symposium on Nonlinear Theory and its Applications (NOLTA 2007), vol. 1, pp. 96-99, 2007.

[17] M.C. Mackey and L. Glass, Science, vol. 197, p. 287, 1977.

[18] R.F. Fox, I.R. Gatland, R. Roy, and G. Vemuri, Phys. Rev. A, vol. 38, p. 5938, 1988.

[19] A. Namajunas, K. Pyragas, and A. Tamasevicius, Phys. Lett. A, vol. 201, p. 42, 1995.

[20] M.-Y. Kim, C. Sramek, A. Uchida, and R. Roy, Phys. Rev. E, vol. 74, p. 016211, 2006.

[21] S. Sano, A. Uchida, S. Yoshimori, and R. Roy, Phys. Rev. E, vol. 75, p. 016207, 2007. 